\title{
An InTERSECTIONAL ANALYSIS OF Visual Media: A Case of Diesel ADVERTISEMENTS
}

\author{
AnTHONY JUSTIN BARNUM \\ Universidade de Cabo Verde \\ and \\ ANNA M. ZAJICEK \\ University of Arkansas
}

This study is intended to advance the application of an intersectional approach that focuses on the simultaneous operation of gender, racelethnicity, and sexuality to the analysis of visual media, such as advertisements. Despite the growing advocacy to systematically include intersectionality in our analyses of people's experiences and identities, on the one hand, and their images/representations, on the other, sociologists still tend to analyze only one of these identities at a time. In this article, we argue that the application of the intersectional approach leads to more complex and adequate understandings of how identities and power relations are constructed in visual media. Towards this end, we conduct an intersectional analysis of Diesel advertisements using the concepts of racialized gender and gendered race, and demonstrate the advantages of an intersectional analysis. In doing so, we hope to provide an illustration of an intersectional analysis of visual media, such as advertisements, which could inform the work of others interested in conducting similar analyses.

\section{Introduction}

In recent years, advertisements have begun to engage diverse social identities to appeal to a wider range of possible consumers (e.g. United Colors of Benton). In doing so, they often construct

Inquiries should be sent to ajbarnum@gmail.com or azajicek@uark.edu. 
race/ethnicity, gender, and sexuality in such a way as to create an illusion that all visible identity markers are equal: "In the eyes of the media, the youths of the world are becoming a single, seamless, soulless target audience" (Kilbourne 1999:41). Ritzer et al. refer to this as the transformation of "nothing into something" (2005:118), which is the result of catering to a homogenized target audience. Specifically, Ritzer et al. (2005:124) state that "the need to transform nothing into something is a response to the homogenization of culture and consumption that accompanies the rise of modern and now postmodern societies." The false world of the advertisement constructs a reality through the equalization of the objects of nature, the body, race/ethnicity, gender, sexuality, and the lack of age and class differences. In this false world, the objects included and excluded become natural in their grouping. Diesel is one of the corporate actors/advertisers that is turning a product of mass consumption (nothing) into something. This becomes problematic when we realize Diesel is participating in a dialectical relationship whereby it is constructing visible identity markers, such as race/ ethnicity, gender, and sexuality, as equals (nothing) and making that equality the substance (something) of Diesel.

Nicholson (1997) addresses the issue of appropriating and consuming multiple identities using the concepts of "resistance" and "oppositional reading." Nicholson (1997:183) says that through the "commodification of 'resistance' . . these types of ads position their products in terms of anti-establishment scenarios, allowing the consumer to purchase a 'resistant' identity along with the product - these ads in effect provide the reader with an 'oppositional reading." The result is that instead of actively participating in a movement through social action that could affect change, individuals actively participate in consumerism that expresses the appropriated ideas of the social movement (Nicholson 1998: 209). One possible reason why we are willing to buy the revolution and wear it instead of living it is the ideal of "consuming difference," which enables us

To theorize how gender and multicultural identities are represented in contemporary advertisements. Our identity guides much 
in our consumption patterns, and the interaction of consumption and identity in a society such as ours signals the power of marketing. In a visual consumer society, our consumer choices reflect our values (Schroeder 2002:10).

Importantly, the construction and the consumption of the difference in advertisements are embedded in complex power relations marked by intersections of race/ethnicity, gender, sexuality, social class, and age. That is, advertisements try to target specific audiences by constructing race/gender/sexuality/class/age appropriate representations of their identities and experiences. Recognizing the complexity of identities, experiences, and representations, intersectional theorists, mostly feminists of color, developed a theoretical perspective that allows us to examine how socially constructed categories of race/ethnicity, class, gender, sexuality and age simultaneously interact to create unique identities and life experiences. In recent years, the intersectional perspective has gained recognition in different disciplines, including social sciences (Collins, 1993) and cultural studies (hooks, 1990, 1992; Morrison, 1992).

Importantly, despite these theoretical developments, very rarely do we try to move beyond one-dimensional analyses of race/ethnicity, or gender, or sexuality. For instance, in an attempt to "facilitate a "media literacy," Nicholson (1998) examines the hegemony of whiteness in advertisements using a 1994 Diesel Jeans and Workwear campaign. Although very valuable, his analysis glosses over the embeddedness of gender and sexuality within whiteness. Yet, white men's and women's positionalities in advertisements are not the same (see also Dines \& Humez, 2003). In fact, even if we do integrate two or more dimensions in our analyses, we still tend to analyze these as separate identities and/or representations (see, for instance, Scodari, 2003). As a result, research focusing on the intersections of two dimensions of inequality such as race/ethnicity and gender (see, for instance, Wlodarz, 2005) is very limited.

The purpose of this study is to analyze advertisements located in the public realm in order to advance an intersectional analysis of visual media. We believe an intersectional approach is necessary to gain a comprehensive and holistic understanding of human 
experiences and identities, including their representations in the media. We also believe that by employing intersectional perspectives we can more effectively challenge and resist the equalization and homogenization of visible identity markers. In fact, the value of intersectional perspective is its unique contribution to the deconstruction of the hegemonic representations of the body in visual media, on the one hand, and its ability to uncover the complexity of meanings embedded in advertisements, on the other.

In this article, we focus on the Diesel advertisement campaign "Nature: Love It While It Lasts" to illustrate and provide a model for an intersectional reading of visual media, such as advertisements. In this context, we pay particular attention to the gendered and sexualized representations of race (gendered race), on the one hand, and to the racialized representations of gender and sexuality (racialized gender), on the other. Throughout, we highlight the advantages of an intersectional analysis. We begin by discussing advertisements and the social construction of meanings.

\section{Advertisements and Symbolic Environments}

In advertisements, human bodies are placed in symbolic environments that shape and reflect cultural identities (Greider \& Garkovich 1994). In this context, we use Greider and Garkovich (1994:1) to talk about landscapes or those "symbolic environments created by human acts of conferring meaning to nature and the environment, of giving the environment definition and form from a particular angle of vision and through a special filter of values and beliefs." When race/ethnicity, gender, and sexuality are placed within a landscape, the ensuing socially constructed environment no longer simply reflects the symbols and meanings we assign to the landscape. Instead, we assign new symbols and meanings to these descriptors and their place within the environment and society at large. These descriptors of cultural identities are seen in the landscapes through the symbols and meanings that are norms of "proper and improper relationships among themselves and between themselves and the physical environment" (Greider \& Garkovich 1994:2). 
Importantly, the descriptors of cultural identities that are placed within a landscape by the constructor or "culture manufacturer" (Horkheimer \& Adorno 1972:124) are not always interpreted by the viewer or not interpreted according to the intent of the culture producer. This is in part due to the fact that cultural identities are defined by the society or subculture that is viewing and interpreting them.

Although changes in the environment may require renegotiation of meanings, in the socially constructed landscape of advertisements, the consumer "has to accept what the culture manufacturers offer" (Horkheimer \& Adorno 1972:124). The symbols and meanings are chosen by the cultural manufacturer and placed inside the landscape but they may or may not be readily understood or noticed by the viewer. In fact, consumers typically connect with a small number of ads that we find to be matching our identities and lifestyle or that perhaps elicit a hidden desire (Schroeder 2002:30).

In this context, "[t]he use value [of nature] is based upon the transformation of nature into culture in the process of consumptive appropriation" (Eder 1996:12). It is this consumptive appropriation that establishes the desired image of the product (Eder 1996:13). Advertisements through appropriation take value from nature, the body, race/ethnicity, gender, social class, age and sexuality and transfer that value to the ideal image. Meaning and value are not necessarily transferred back to nature, the body, race/ethnicity, gender, social class, age or sexuality.

At the same time, advertisers attach notions of the good and the beautiful inherent in the representations of nature and Otherness to their products. Thus the distinction between product and nature or the socially constructed reality within the advertisement becomes blurred as the meanings behind nature, the body, and the product are transferred back and forth. The fluidity of symbolic meanings in this dialectic forms a new socially constructed meaning, which is transmitted into the public realm, a meaning of egalitarian sameness. In this context, "[n]ature and the environment are valued only insofar as they can improve the profitability of the advertiser" (Frith 1995:185). This use value of nature and the environment in advertising is most visible in its use as a conduit that provides a 
pathway for symbolic meanings to be transferred from nature to the product.

According to Greider and Garkovich (1994:5), the meanings and symbols of a socially constructed environment, including nature, are imposed on less powerful groups. Those groups upon which meanings and symbols are placed can be racial, ethnic, gendered, sexualized, etc. Greider and Garkovich (1994:5) maintain that "human actors ... use culture as a resource to construct symbols and meanings that define nature, the environment, and human-environment relationships and ... [that] power is used to impose these social constructions on other groups." In short, the social construction of the environment and the human body occurs through hegemony. The complexity of the hegemonic images and symbols can be deconstructed using the perspective of intersectionality.

\section{Intersectionality Perspective}

The discourse related to the intersections of oppressions such as race/ethnicity, social class, gender and sexuality has emerged across the social sciences as an appropriate theoretical framework shaping one's research agenda. Hence, an increasing number of scholars recognize how the intersections of these oppressions shape people's experiences (Crenshaw 1991; Collins 2000; Andersen 2005; McCall 2005) and have cautioned against traditional, simplistic, one-dimensional analytical conceptualizations of oppressions (Collins 2000).

Intersectionality, a perspective developed to account for the complexity of human identities and experiences (Essed, 1990, 1991), sensitizes us to the importance of examining the articulation of various dimensions of difference/otherness in order to discern how they become organized into what Collins (2000) calls a "matrix of domination." We define intersectionality as an integrated approach that identifies and examines the multiple forms of inequality. Intersectionality is used to analyze the interactions of inequalities as well as the connections and the co-construction of various aspects of identity, such as gender, race, and sexuality, 
resulting in complex representations of sexualized and racialized bodies. At the most basic level, intersectionality perspectives "trace the consequences of class, gender, race, affectional preferences, global location for lived experience, group standpoints and relations among women [and men]" (Lengermann and Brantley 2004:349). Methodologically, intersectionality is a multi-method approach that can be used in qualitative as well as quantitative studies aiming at understanding the various ways in which systems of inequality as well as dimensions of identity intersect.

Intersectionality in relation to advertising allows us to understand how race/ethnicity, sexuality, and gender while being "at once historically and geographically specific ... are also transnational, encompassing diasporic groups and crossing traditional geographic boundaries" (Zinn \& Dill 1996). More specifically, intersectionality calls for a different, more complex and more holistic, reading of signs and symbolic orders. If we ignore the different dimensions of diversity, we perceive "differences as the product of groupspecific values and expressions of a normative center" (Zinn \& Dill 1996).

As an integrated and holistic approach, while intersectionality has several advantages over one-dimensional approaches, it also presents researchers with a set of unique challenges. With regard to its advantages, this perspective allows "a conceptualization of [social inequalities] that attempts to capture both structure and dynamic consequences of the interaction between two or more axes of subordination" (Patel 2001:9). Second, it also allows us to view people's identities as multifaceted and context specific, wherein the meaning of people's locations and identities as well as their salience are the product of history, culture, and society (Landry 2006). Third, depending on our social location in a given society, certain aspects of our identity, like race, gender, or sexuality, may protect us from discrimination while its other dimensions may lead to discrimination and other human rights violations (Crooms 1997).

To account for the interactions of race/ethnicity and gender, intersectionality theorists use the concept of gendered racism. Philomena Essed (2001:3) introduces the concept of gendered racism as follows: 
Gendered racism shapes the allocation of resources along racially and ethnically ascribed understandings of masculinity and femininity as well as along gendered forms of race and ethnic discrimination...According to the different ideologies and histories in relation to specific ethnic groups women are sexualized in different ways. European common sense considers African women generally as promiscuous, Asian women as passive and exotic, and women in Islamic cultures as super-exploited.

In this paper, we build on Essed's ideas and use the concepts of racialized gender and gendered race. The concept of racialized gender denotes the idea that gender ideologies, expectations, and norms operate differently across diverse racial/ethnic groups (e.g. African-American women vs. white women). In doing so, this concept deconstructs the idea that women are a homogenous category. For instance, although African American women and white women are women, historically, the African American women have been expected to be independent and strong while white women have been expected to be dependent and submissive. To put this differently, the racialized gender concept emphasizes racial/ethnic variations among people of the same gender. In contrast, the concept of gendered race denotes the existence of gender differences within the same racial/ethnic group. That is, the situation wherein the members of the same racial/ethnic group (e.g. white men and white women) experience different expectations, ideologies, and norms based on their gender. As such, the concept of racialized gender challenges the validity of treating racial/ethnic groups (i.e., white people) as unitary categories.

Regarding the unique challenges this perspective presents to researchers, one of the most salient challenges involves how to manage the complexity of an intersectional analysis. Addressing this challenge, McCall discusses three methodological approaches to managing the complexity of intersectional analyses. The first approach is called "anti-categorical complexity" (2005:1776-1780). Scholars using this approach focus on deconstructing existing systems of categorization in order to expose the arbitrary nature of such systems. The anti-categorical approach begins with identifying different ways in which social scientists construct and use unified/ 
homogenized social categories such as women, men, homosexuals, heterosexuals. Next, the anti-categorical analysis works its way outward to expose how this categorical homogeneity could only be constructed if one glossed over the influences of gender, race, and other vectors of inequality.

The second approach is intra-categorical complexity. The only difference between intra and anti-categorical approaches is the methodology used. Scholars using the intra-categorical approach place the subject at the intersection of a single dimension of multiple categories. For example, middle class African-American women are placed at the intersection of multiple categories (race, class, gender) and then studied in depth as a single social group whose experiences are defined by the unique intersection of these categories (McCall 2005:1781).

The third approach is inter-categorical. This approach focuses on "the complexity of [the] relationship between multiple social groups within and across analytical categories and not on complexities within single social groups and/or categories" (McCall 2005: 1786). This approach differs from two previous approaches because it involves comparing and contrasting the multiple locations of selected social groups. This process is very complicated as it involves comparative investigation of multiple categories of multiple social groups. McCall gives an example of inter-categorical approach by first incorporating gender as an analytical category. Such analysis assumes two groups: men and women. If class is incorporated, the gender must be cross-classified with class - working, middle and upper. Thus, six groups are created. If race and ethnicity are also included, then the number of group expands to twelve. Similarly there may be other categories like education or nationality, which may be included while analyzing gender. In sum, the inter-categorical method of intersectionality analyzes the intersection of the sets of dimensions of multiple categories, thereby exposing complex matrices of advantage and disadvantage (McCall 2005:1786-1787).

To manage the complexity of our intersectional study, we adopt the inter-categorical approach. Several reasons underlie this decision. First, in line with the inter-categorical approach, we recognize the relationships of inequality among groups constituted 
by interacting inequalities (see McCall 2005:1784-1785). Next, we assume that the individuals presented in the ads are members of and/or represent specific social groups defined mainly by the intersections of race/ethnicity, gender, age and social class. We also assume that the ways in which the individuals are portrayed signify something meaningful about their sexuality. Finally, in order to arrive at our conclusions and interpretations, we engaged in a comparative analysis of how individuals representing each group are portrayed and what the ads imply about the relationships among them. Importantly, given the fact that an inter-categorical analysis involves comparing groups or categories defined by differences, in our examination of Diesel ads we could not compare and contrast individuals based on their social class and age, as these two dimensions do not vary in the ads.

In the next section, we discuss our method more in depth and note the limitations underlying our categorization/identification of individuals depicted in the ads.

\section{Methods}

To collect information-rich cases for our study, we used purposeful sampling (Patton 1987: 51-52). We chose to use the 2004 Diesel clothing campaign entitled "Nature: Love It While It Lasts" as the data source for this paper due to their rich layering of visual identity markers. In order to gain access to this advertisement campaign, we relied upon the Diesel website (Diesel). Our sample is a critical case "that can make a point dramatically" (Patton 1987:54) and is "particularly information-rich or enlightening" (Crabtree 1992:30) specifically since our sample contains multiple and simultaneous points of intersection of gender, race/ethnicity, and sexuality.

The accessibility of media and thus of knowledge, limits the reach of advertisements to their audience. Diesel advertisements are reserved for a select audience that has access to internet, which we relied on, and the leisure time to peruse their site and to the few magazines they utilize to expose themselves. These advertisements are usually designated for an upper or upper-middle class that has 
the disposable income necessary to subscribe to magazines and to the internet bringing them greater access to the arts and popular culture. ${ }^{1}$ This restriction of cultural capital results in a lack of access for marginalized groups and a lack of representation of those groups. Given this, we assume that the upper-social class discourse is embedded in Diesel advertisements.

We conducted content and structuralist/semiotic analysis (Ball \& Smith, 1992; Williamson, 1978) of these documents, linked them to intersectionality, and analyzed them in regard to dominant ideologies, such as white heterosexual capitalist patriarchy. According to Ball and Smith, (1992:31) a structuralist analysis enables us to "arrive at a fuller appreciation of the visual representation by relating it to other social and cultural arrangements." Moreover, "[b]y treating the advertising image as a 'text,' semiotic analyses concentrate on the relationships between the ads' internal meaning structures as they relate to the larger cultural codes shared by viewers" (Shields 1990:26). The advertisement is not only constructed by a sociocultural group, but is reified by it (Greider \& Garkovich 1994:6). Thus, the use of advertisements allows us to "read aspects of the social world through them" (Mason 2002:106). Mason argues, "literal 'readings' of visual data and documents should not extend to treating them as though they are direct representations or reflections of 'reality' or straightforward 'factual records"' (2002:107-108). In this context, by examining the intersectional themes in Diesel advertisements, our analysis will show how to use an intersectionality perspective to deconstruct visual images and their power-laden discourses.

We then identified the intersectionalities of race/ethnicity, gender, and sexuality using a priori codes and merging themes (Charmaz 2001). We analyzed our data using Mason's (2002:149) three methods for reading data: the literal, interpretive, and reflexive. This holistic reading of data directed us in a latent content analysis (Glassner \& Corzine, 1982) or the search for the "deep

\footnotetext{
${ }^{1}$ Over the past five years, several magazines and newspapers (e.g., Time, Financial Times) published articles discussing luxury fashion brands. Diesel is often mentioned in this context (see, for instance Dyk et al. 2008; Michaels 2008; Vesilind et al. 2005; Businessline 2007; Michaels 2007).
} 
structural meaning conveyed" (Berg 1995:176). When we critically analyze advertisements we are able to visualize the intersectional politics of domination (hooks 1992:5) as expressed through the visual matrices of race/ethnicity, gender, and sexuality.

A caveat must be made before we turn to our analysis. In our analysis, we categorized people portrayed in advertisements based on our visual perceptions. For instance, with regard to race/ethnicity we used the skin color as its representation. The skin color of the individuals is the primary means we have for ascribing race/ethnicity of actors even though these measures are not measures of their subjective identities. Thus, in doing so, we imposed categories on social life, which "is considered too irreducibly complex-overflowing with multiple and fluid determinations of both subjects and structures - to make fixed categories anything but simplifying social fictions that produce inequalities in the process of producing differences" (McCall 2005: 1773). Since we are not able to determine the heritage of the models or their subjective definitions of their racial/ethnic identity, we feel that by relying on loose definitions of group membership we perhaps further stereotype individuals. Even though we see this as a weakness, we realize that this is how a majority of individuals interpret race/ethnicity by basing it solely upon the visual aspects of an individual. Race/ethnicity as a social construction is most often based upon "perceived" differences. Thus we assume that our construction is a valid approximation of how race/ethnicity can be interpreted.

\section{Case Study: An Intersectional Analysis of Diesel Advertisements}

\section{Situating Intersectionalities}

In order to understand the interactions of race/ethnicity, class, gender, and sexuality, we first must understand the body, especially as it is presented within advertisements. According to Bakhtin (1968), ritual spectacles are best explained by looking at carnival, where traditional hierarchies and the authorities that go with them are reinvented. In the world of carnival, power structures are symbolically reversed. Structures of social interaction, impossible at 
other times, become common with individuals acting as a group. This world of carnival presented within the Diesel advertisements reinvents the structures of social interaction by allowing the various interactions of race/ethnicity, class, gender, and sexuality to coexist simultaneously while ignoring traditional hierarchies. It is the action of carnival that works to maintain hegemony by allowing a small symbolic counter-hegemony. This is what Diesel appears to accomplish. "From the standpoint of white supremacist capitalist patriarchy, the hope is that desires for the 'primitive' or fantasies about the Other can be continually exploited . . . in a manner that reinscribes and maintains the status quo" (hooks 1992:22). The carnivalesque body thus becomes the site where the structures of social interaction cease to follow traditional rules and hierarchies and begin to exhibit the primitive fantasies of the Other.

\section{Social Class and Nature}

Due to the status symbolism of Diesel clothing and the placement of advertisements in trendy fashion magazines (e.g. The Face), one expects the portrayal of individuals within the Diesel campaign to imply, or at least not call into question, that they are members of a privileged class. Our analysis of the ads confirmed this expectation, leading as to believe that the lack of representation of less privileged social classes becomes yet another factor buttressing the sign value of Diesel clothing. In doing so, the campaign goes beyond specifically targeting the advertisements to only those with the capital to conspicuously consume Diesel clothing. As Nicholson (1997:178) puts it, the hegemony present in advertising allows us to "see how this dominant class addresses their particular target audiences, and also what these ads may be saying - sometimes through exclusion - to members of the nontarget audience."

The exclusion that Nicholson refers to also occurs via the construction of the relationship between people in the ads and nature. Specifically, people from lower socioeconomic backgrounds are often denied access to nature, especially the diverse and exotic natural environment portrayed in the ads, due to lack of transpor- 
tation and financial resources to journey to far away lands. ${ }^{2}$ The exclusion of non-target audience is thus both recreated and fortified within the Diesel advertisement, where once again those of lesser socioeconomic status are denied entry into this Diesel paradise. As we will discuss next, this highly classist image interacts in complex ways with the racialized, gendered, and (hetero) sexualized representations of nature, "denying access" to many "others."3

\section{Gendered Race/Ethnicity}

When conceptualizing intersectionalities it helps to focus attention on categories or anti-categories. For the purposes of this paper, we use gendered race and racialized gender to obtain a clearer demarcation zone whereby we neutralize certain identity markers in order to understand how those neutralized markers affect other markers simultaneously. In the Diesel campaign "Nature: Love It While It Lasts," it is upon the background of nature that the body is placed and upon the body that race/ethnicity, gender, and sexuality are placed creating a palimpsest and a pastiche of meanings, where identity markers are in constant change and fluidity. Gendered race or "controlling" for race to look at the effects of gender within a race category is a means to understand the matrices of domination (Collins 2000) between different genders and how genders are experienced, acted out, and portrayed within the confines of a racialized boundary. Sexuality will also be looked at within this semi controlled anti-categorical manipulation. Diesel takes nature and reconstructs it as an androgynous being with gendered aspects.

\footnotetext{
${ }^{2}$ Another aspect of the classist undercurrent of the Diesel advertisement is in the educational background of the target audience. Being directed at the middle and upper classes also means targeting a more educated audience. Usually those with higher educational background are more concerned about environmental issues, which is why "Nature: Love It While It Lasts" appeals to them. This direction towards a more educated clientele is one more way that Diesel can be elitist.

${ }^{3}$ Diesel advertisements become a representation of a landscape where a class of people with relatively more buying power interact with nature. The advertisement becomes not only a set of instructions on how a certain class interacts with nature, but also becomes a goal for lower classes to attain.
} 
In one image, there are two individuals, one female and one male of possible Asian descent. In the style of Georgia O'Keefe, flowers are used to represent sexual organs. The flower held by the Asian man is yonic in meaning with its pink color and the man's fingers preparing to enter the center. However, the flower held by the Asian woman is phallic in representation stretching toward her open and waiting mouth. These two individuals encourage a heterosexual reading of the ad. The genders of the two flowers are contrasted against the two individuals who though female and male share similar traits. Similar skin color, neither black nor white, hair color, black but straight, and the similar style of Diesel brand eyeglasses that they are wearing creates an ideal of androgyny and somewhat ambivalent racial identity for the individuals in stark contrast to the hyper-gendering of the flowers representing nature. This role of androgyny reaffirms the controlling image of gender for Asian women and men, who are typically seen as less masculine and more feminine. The use of flowers to represent both female and male lends itself once more to the androgynous image of nature. It must be noted that although androgyny is being portrayed here it is still heterosexual due to the hyper-gendering of the flowers and the pairing of female with male and male with female. Thus in any certain matrices of domination, while some identity markers are neutralized, others are exaggerated, rendering impossible the equalizing of all identity markers within a given point of intersectionality.

Once again treating the idea of gendered race we take into consideration the portrayal of black men and black women. Hooks talks about the "young black male body" and how it "is seen as epitomizing this promise of wildness, of unlimited physical prowess and unbridled eroticism" (1992:34). A different intersectionality of race/ethnicity and gender can be seen in how the black men's and black women's bodies are presented in the Diesel ads. Specifically, here the black men's body is light-skinned as compared to the black women's body that is represented as both dark-skinned and light-skinned. Finally, the intersectionality of race/ethnicity, gender, and sexual orientation is also present in Diesel advertisements. For instance, the black men's bodies found in the advertisements are 
always represented as not participating in bisexualities or homosexualities. Heterosexuality is implied and even concentrated in another image where a black man is seen performing heterosexuality with a white woman and a female gendered nature.

The Diesel advertising campaign works hard to prevent us from being able to look at any given image and see a representation of gendered race. Individuals within the advertisements are almost always paired with members of different genders and races to avoid images of segregation and a basis for comparison. Another advertisement presents us with just such an image of a black woman and a white man. The black woman is seen with a phallic cactus between her thighs and the white man is seen biting into the end of a cactus representative of a female breast. In this advertisement both humans and nature are seen as gendered and heterosexual. But once again, the use of cacti to represent both the female and the male create androgynous nature. This androgynous identity is counterbalanced once again with a functional hegemony of gender, sexuality, and race/ethnicity.

This androgynous nature is removed from the advertisement except for the two gendered cacti. Both the black woman and the white man are also wearing black, mirrored sunglasses further separating them from nature and each other. Unable to see they are deprived of a means of interaction with not only each other but also with the gendered and sexed nature. This hiding of their eyes from the audience also objectifies the models depersonalizing them and emphasizing their use value as bodies. The separation caused by the use of the sunglasses is also countered by the fact that the bodies of the black woman and white man seem to be touching, an act almost nonexistent within the advertisements. When looked at closely, this touching becomes much more than simple contact. The black woman is supporting the white male who is leaning back against her. This introduces into our other category of racialized gender.

\section{Racialized Gender}

Racialized gender controls for gender to look at the differences of race upon the said gender. If we were to replace the black woman 
in one of the advertisements with a white woman, the meaning would change. If a white woman is supporting a white man, one level of intersectionality is removed as race becomes a controlled commonality of experience. To get a better understanding of racialized gender we must look at several different advertisements within this campaign. Taking the women within the advertisements who are bound we can address the question of racialized gender.

"Critical researchers see advertisements as reflecting the dominant ideologies of the society that produces them ... [thus] the entire text ... has the ability to reflect dominant cultural discourses about such things as gender, race, ethnicity" (Frith 1995:187). Within this particular advertising campaign, Diesel presents women, regardless of race/ethnicity, in more submissive positions than men. There are four examples of women or the woman's body tied and constrained by nature. The first woman constrained is the dark skinned black female entwined by a snake. The other three examples are of white women constrained with plants or bound with vines.

In another advertisement, the dark skinned black female body symbolizes betrayal through the symbolism of the snake coiled around her body as she lies upon a tree branch. The biblical allegory developed here is that of Eve and the serpent betraying the race of man, and "the black female body . . . is ... depicted as a site of betrayal" (hooks 1995:16-17). This is contrasted with the white female bodies. In Diesel's advertisements, white female bodies are restrained differently than the black female body. The white females are restrained in the act of sexual bondage. In this type of restraint there is no betrayal depicted, simply a naiveté and the idea of a stolen virtue not accorded the black female body: "the white female ... symbolizes innocence and virtue" (hooks 1995:17).

Revisiting gendered race, the contrast between bound white women and bound white men is again visible; there is only one white man who is restricted in such a manner. The only male partially constrained is draped with a vine. However, his bondage is only partial, as opposed to the full constriction of two of the white women, one light skinned and one dark skinned, and the dark skinned black woman. Both women and men, regardless of 
race/ethnicity, are dominating nature within this advertisement as members of a generic humanity, yet women are still dominated by patriarchal positioning of women as subdued and constrained. Women, bound by nature, are tied more closely to nature so that they can be dominated by patriarchal ideology, and are more likely to have restricted freedom of movement and more availability to male readers viewing the advertisement.

The sexual roles portrayed within the advertisement provide another point at which to examine racialized gender. Another advertisement contains the subject of a black woman. She is placed upon dark nature, but pulled out of the background by a lighter colored tree and her Diesel attire. This dark skinned black female body is exoticized not only through the intersections of race, ethnicity, and gender, but also with tropical birds creating a link between exotic destination and exotic individual. She is also sexualized by the emphasis placed upon her butt by the bright blue skirt that draws the viewers' attention. Hooks writes in her essay "Selling Hot Pussy" that "in the sexual iconography of the traditional black pornographic imagination, the protruding butt is seen as an indication of a heightened sexuality" (1992:63). This highlighting of the butt is a sexualized stereotype of black female sexuality in a white supremacist patriarchal context. This black female role is contrasted to the white female role. Here we see a white woman with partially open mouth and moist lips approaching a flower that she is caressing with her hand. Once again the combination and positioning of the female model and the phallic symbolism of the flower, complete with the image of veins, guide the viewer into conceptualizing the act of fellatio.

The white flesh tones of the flowers, plants, and of the shell enhance the viewers' ability to read the objects as human body parts, specifically white sexual organs. Anthropomorphizing plant forms within the Diesel campaign is the means by which nature is given a sexual identity by the human participants. When sex is diminished to just the sexual organs as the only needed parts of the body the sexual objectification of the body is achieved. This also solidifies the use value of nature in the human-nature relationship. 
Sexuality, Race/Ethnicity, and Gender

The inclusion of male and female homosexuality is unique in the ads as the sexualities within the advertisements are subtle, allowing the viewer to overlook them if they so choose (Clark 1995:144). One advertisement presents a white cowboy riding a tree branch. This advertisement elicits a homoerotic interpretation of the gay cowboy riding the phallus. This possible interpretation allows advertisers to target multiple audiences using the same advertisement, which leads them to achieve a more global perspective in the eye of the consumer who sees the same advertisement everywhere, not altered for different consumer contexts.

The Diesel advertisements portrayal of multiple sexualities lends itself to the "fluid nature" of sexuality and "the possibility that sexual nature can be stable for some and changeable for others" (Zajicek et al. 2001: 238). This fluidity can be seen in the combination of partners within the advertisements. The continuation from one individual to the next uses nature to unite and tie together actors within the advertisements. It must be noted that for the most part all individuals are portraying sexualities with nature as a partner, thus depending on whether the particular nature is gendered female or male determines whether the sexuality is hetero, homo, or bisexual. For example, in one image, the tree on the left side is simultaneously engaged with four individuals, a dark skinned black woman and two dark skinned white women and a dark skinned white man. The gendering of this tree as predominantly male allows for a fluidity of sexuality since it is engaged with members of both genders. The use of lighting and color within this advertisement also places greater emphasis on those individuals who are white or lighter skinned. Due to the use of a dark nature as background, those individuals who are lighter skinned or who are wearing white or light clothing stand out from the background, while those with darker skin and darker clothes blend in and disappear. Another advertisement offers another example of sexual fluidity, where a dark skinned white possibly Hispanic woman and light skinned black man are engaged with two parts of the giant Venus flytrap. The Venus flytrap is gendered female, with its representation as the female sexual organs. 
The sexual fluidity of the plant is implied by "her" simultaneous engagement with both genders, allowing the sexual act to be read as both heterosexual for the black male and as bisexual for the white female. This inclusion of multiple fluid sexualities is inseparable from the fluidity of race/ethnicity and gender. By linking all individual actors and all sexualities through the sexual partner of nature all identity markers are rendered equal.

\section{Conclusion}

The purpose of this research was to conduct an intersectional case study of advertisements located in the public realm to illustrate the principles and advantages of an intersectional analysis. In what follows, we summarize these and note some advantages of engaging in an intersectional analysis.

Within the series of Diesel advertisements "Nature: Love It While It Lasts," a variety of races and ethnicities are represented, all equally removed and isolated from distinct cultures and placed within an alternate world of environmental purity where the racially and ethnically diverse individuals are all equally "cultureless" (Perry 2001), yet still maintain their ethnicity as a sign of the Other. This use of ethnicity as symbolic of the Other is no more than a reduction of ethnicity to physical characteristics that are used to spice up white patriarchal capitalism in the guise of an advertisement campaign (hooks 1992:21). This blending of marked (the Other) and unmarked (the norm) (Brekhus 1998) results in a construction of a social world where the problems of lived experiences are difficult to grasp as they are all equated with culturelessness and normalized into non-existence.

Working from Perry's idea of the culturelessness of whiteness, one can interpret the Diesel campaign "Nature: Love It While It Lasts" as reproducing the Other alongside the norm of whiteness. However, in reproducing the Other (race, ethnicity, gender, and sexuality) as the spice for white mainstream culture, whiteness is being imposed upon the Other. Importantly, this whiteness is not just whiteness but also heterosexuality, maleness, and Westerness. In short, it imposes normness. By acknowledging other iden- 
tity markers, we are able to recognize a major fault with Perry's work - not seeing whiteness as sexualized and gendered. From an intersectional perspective, otherness must also be understood as being many different social constructions depending on the Other being constructed as (heterosexual) white women, black women, black men, Asian women, and Asian men.

Diesel does this within a context of a fantasy nature that is usually linked to the carnivalesque body of Bakhtin. Yet, in this advertisement campaign, the classical body with carnivalesque characteristics is also embedded in nature. This use of nature decontextualizes culturlessness and makes it more appealing and acceptable to the viewing audience and mainstream culture. Diesel is in essence creating a culture of the cultureless. This is done by separating the spice or social markers of Otherness and reassembling them in a pastiche of intersectional identity markers, which we analyzed as gender race and racialized gender. These intersectional identity markers are placed upon a pastiche of nature and the body.

The aspects of race, ethnicity, gender, and sexuality that are emphasized in the Diesel campaign "Nature: Love It While It Lasts" construct postmodern identities that are composed of fractured multiplicities. To accomplish this, first, the most exotic, recognizable, and luscious parts of nature are placed to form the perfect landscape to house the exotic of the body. Next, the exotic is taken from the context of a carnivalesque body and placed upon a classical body that is embedded in a nature illustrating the carnivalesque qualities of the body through such actions as sexual embeds. The exotics of race, ethnicity, gender, and sexuality are removed from the carnivalesque, placed upon the classical, and then embedded in the carnivalesque. Nature serves to insulate the shock value of the separation of the carnivalesque from nature in this landscape.

Nature is almost always aligned with the feminine and the exotic, with minority women being presented as closer to nature. This contradicts the idea of a separation between nature and culture, where the exotic is defined as culture and whiteness is defined as culturelessness. Instead, it is the exotic separated from culture and decontextualized and used as the spice for mainstream white culture 
that becomes representative of culturlessness. Hooks (1992:31) sums this up when she writes that "the commodification of difference promotes paradigms of consumption wherein whatever difference the Other inhabits is eradicated ... by a consumer cannibalism that not only displaces the Other but denies the significance of the Other's history through a process of decontextualization." In this way, the Diesel campaign achieves an equality among race, ethnicity, gender, and sexuality but at the expense of taking the perceived culturelessness of whiteness and imposing it upon all groups and identities represented in the advertisements, which, in turn, help to spread an image of "culturelessness" to consuming viewers.

Both authors have often heard the argument that the more sociologists deal with race and, by extension, other identity markers such as ethnicity, gender, and sexuality, the more we solidify differences in humanity and thus maintain conflict. It is our contention that when we ignore these identity markers, and especially their intersections, we are in effect creating either culturelessness where every aspect of identity is defined in relation to the norm, or we simplify complex identities to a single dimension. This inability to see the interactions of identity markers or the refusal to deal with them through meaningful discussion ignores the importance these markers have in our lives. However constructed the conflict may be among these identity markers, by not addressing them as important aspects of relations of domination and of the social fabric, not only do we oversimplify the complexity of identities but we also fail to ameliorate and/or alleviate tension between marked and unmarked groups and between multiple identity markers within single individuals.

The use of intersectionality to allow the multiple boundary crossings of race, ethnicity, gender, and sexuality provides a more holistic approach to understanding the subtle modern-day discriminations that occur within advertising and that become part of consumer culture. Once we understand these matrices of domination we can more actively participate in alleviating the suffering that they cause on an individual basis. Although in this research we used the intersectionality perspective to analyze a particular series of advertisements by the Diesel company, this perspective can be 
applied to other visual media, including advertising campaigns, to see how complex intersectionalities are constructed and how they continually enter our consciousness.

In sum, our goal was to show the value of intersectional perspective in deconstruction of the hegemonic representations of the body in visual media. Toward this end, we conducted intersectional analyses of the politics of domination present in Diesel advertisements. We believe that intersectional analysis provides a resource for shifting our perceptions of the meanings embedded in advertisements. As such, it also has potential for alternative pedagogy and knowledge creation that are connected to a multi-dimensional social change.

\section{Appendix}

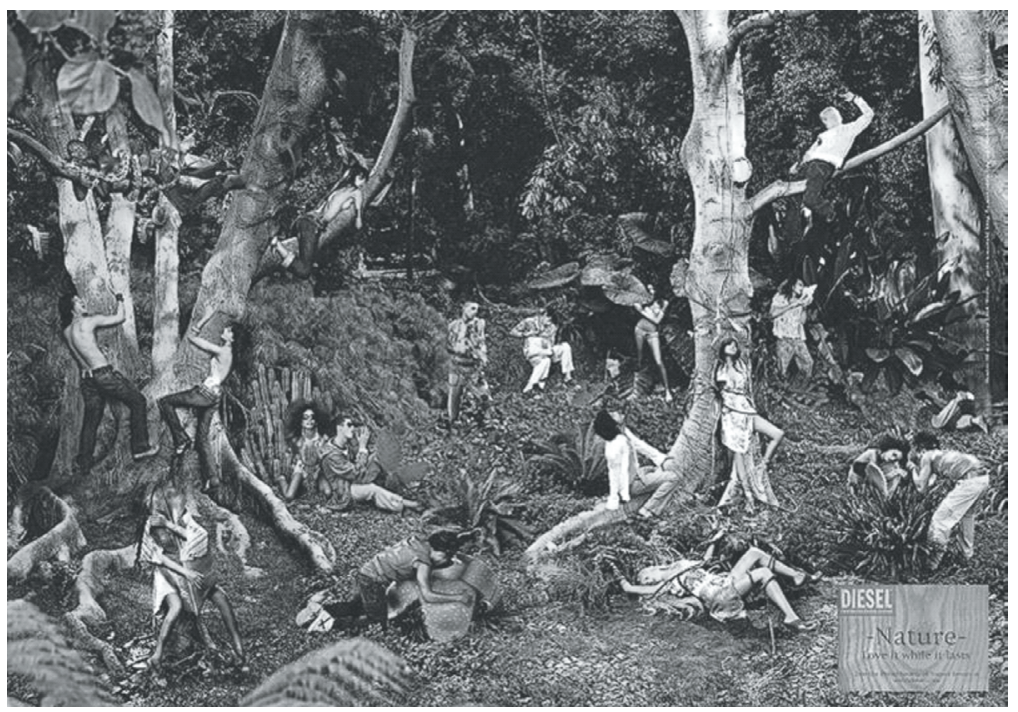

Editor's Note: Although Diesel's Ad Campaign, "Nature: Love It While It Lasts," is no longer available through Diesel's website, there are a few websites that have reproduced some of the images. URL's include:

http://www.xihalife.com/bbs/photos/sexygirls/1339.htm http://jozworld.club.fr/diesel_00.html http://towleroad.typepad.com/towleroad/2004/04/the_garden_of_d.html 


\section{References}

Andersen, Margaret. 2005. Thinking about women: A quarter century's view. Gender and society 19:437-455.

Bakhtin, Mikhail. 1968. Rabelais and his world. trans. Helene Iswolsky. Cambridge: The M.I.T. Press.

Ball, M. S., \& Smith, G. W. H. (1992). Analyzing visual data. Newbury Park, CA: Sage Publications.

Collins, P. H. (1993). Toward a new vision: Race, class, and gender as categories of analysis and connection. Race, Sex \& Class, 1(1), 25-45.

Dines, G., \& Humez, J. M. (2003). Gender, race, and class in media: a text-reader (2nd ed.). Thousand Oaks: Sage Publications.

Essed, P. (1990). Everyday racism: reports from women of two cultures (1st ed.). Claremont, CA: Hunter House.

Essed, P. (1991). Understanding everyday racism: an interdisciplinary theory. Newbury Park: Sage Publications.

Glassner, B., \& Corzine, J. (1982). Library Research as Fieldwork: A Strategy for Qualitative Content Analysis. Sociology and Social Research, 66, 305-319.

hooks, b. (1990). Yearning: race, gender, and cultural politics (1st ed.). Toronto, Ont., Canada: Between-the-Lines.

hooks, b. (1992). Black looks: race and representation. Boston, MA: South End Press.

Kennelly, I. (2004). The Intermingling of Race, Class, and Gender: A Theoretical Commencement. Unpublished manuscript: Unpublished Manuscript. Department of Sociology. George Washington University.

Landry, B. (2006). Race, gender and class : theory and methods of analysis. Upper Saddle River, N.J.: Prentice Hall.

McCall, L. (2005). The Complexity of Intersectionality. Signs: Journal of Women in Culture and Society, 30(3), 1771-1800.

Morrison, T. (1992). Playing in the dark: Whiteness and the literary imagination. Cambridge, MA: Harvard University Press.

Scodari, C. (2003). "No Politics Here": Age and Gender in Soap Opera in "Cyberfandom". In G. Dines \& J. M. Humez (Eds.), Gender, race, and class in media: a text-reader (pp. 497-506). Thousand Oaks: Sage Publications.

Williamson, J. (1978). Decoding advertisements: ideology and meaning in advertising. London: Boyars : Distributed by Calder and Boyars.

Wlodarz, J. (2005). Maximum Insecurity: Genre Trouble and Closet Erotics in and out of HBO's Oz. Camera Obscura, 20(58), 58-105. 